PROCEEDINGS OF THE

AMERICAN MATHEMATICAL SOCIETY

Volume 138, Number 3, March 2010, Pages 1121-112

S 0002-9939(09)10087-4

Article electronically published on October 20, 2009

\title{
THE CONVERGENCE OF THE MINIMAL POSITIVE FUNDAMENTAL SOLUTIONS UNDER RICCI FLOW
}

\author{
SHIJIN ZHANG
}

(Communicated by Richard A. Wentworth)

\begin{abstract}
In an unpublished paper, Hsu gives a proof of the convergence of the fundamental solutions. Since we had a problem understanding Hsu's paper, in this paper we give a detailed proof of the convergence of the minimal positive fundamental solutions of the conjugate heat equation on complete non-compact manifolds under the Cheeger-Gromov convergence of Ricci flows.
\end{abstract}

\section{INTRODUCTION}

In this article we consider the Ricci flow

$$
\frac{\partial}{\partial t} g_{i j}=-2 R_{i j}
$$

on $M \times[0, T](T<\infty)$, where $\left(M, g_{i j}(t)\right), t \in[0, T]$, are complete non-compact manifolds with bounded curvature and $R_{i j}(t)$ denotes the Ricci curvature of $g_{i j}(t)$, $\operatorname{dim} M=n$, and $u$ is a solution to the conjugate heat equation

$$
-\frac{\partial}{\partial t} u-\triangle^{t} u+R(t) u=0
$$

where $\triangle^{t}$ denotes the Laplacian operator with respect to a solution $g(t)$ to $(0.1)$ and $R(t)$ is the scalar curvature of $g(t)$. In this article, for simplicity, we will write $\triangle^{t}$ as $\triangle$ and $R$ instead of $R(t)$. Notice that if $g(t)$ is defined on an interval $[0, T]$ and we let $\tau=T-t$, then (0.2) defines a strictly parabolic equation on $M$ with respect to $\tau \in[0, T]$, i.e.,

$$
\frac{\partial}{\partial \tau} u-\triangle u+R u=0
$$

We will prove the following theorem.

Theorem 0.1. Let $\left(M_{k}, g_{k}(t), x_{k}\right)$ be a sequence of pointed Ricci flow (0.1) where each $M_{k}$ is a non-compact manifold with bounded curvature such that $\left(M_{k}, g_{k}(t)\right)$ is complete for $t \in[0, T]$. Suppose

$$
\left|R m_{k}\right|(x, t) \leq K, \forall(x, t) \in M_{k} \times[0, T], k \in \mathbb{Z}^{+},
$$

Received by the editors February 7, 2009, and, in revised form, June 7, 2009, and June 17, 2009 .

2000 Mathematics Subject Classification. Primary 58J35, 53C44; Secondary 35K05.

Key words and phrases. Ricci flow, conjugate heat equation, minimal positive fundamental solutions.

The author was supported by the China Scholarship Council.

(C)2009 American Mathematical Society 
for some constant $K>0$ and

$$
\left\{\left(M_{k}, g_{k}(t), x_{k}\right)\right\}, t \in[0, T],
$$

converges in the $C^{\infty}$ pointed Cheeger-Gromov sense to a smooth solution of Ricci flow (0.1),

$$
\left(M_{\infty}, g_{\infty}(t), p\right), t \in[0, T] .
$$

That is, there exists an exhaustion $\left\{U_{k}\right\}_{k \in N}$ of $M_{\infty}$ by open sets with $p \in U_{k}$ and a sequence of diffeomorphisms $\Phi_{k}$ of $U_{k}$ to open sets $V_{k}$ of $M_{k}$ such that $\Phi_{k}(p)=$ $x_{k}, \forall k \in \mathbb{Z}^{+}$, and the pull-back metric $\Phi_{k}^{*}\left(g_{k}\right)$ converges $C^{\infty}$ to $g_{\infty}$ uniformly on compact subsets in $M_{\infty} \times[0, T]$ as $k \rightarrow \infty$.

Define the function $u_{k}$ on $M_{k} \times[0, T)$ to be the minimal positive fundamental solution of the conjugate heat equation limiting to the $\delta$-function centered at $x_{k}$ as time approaches $T$; i.e., $u_{k}$ is the minimal positive solution to (0.2) and

$$
\lim _{t \nearrow T} u_{k}(\cdot, t)=\delta_{x_{k}} .
$$

Then $\Phi_{k}^{*}\left(u_{k}\right)$ subconverges uniformly on every compact subset of $M_{\infty} \times(0, T)$ to the minimal positive fundamental solution $u$ of the conjugate heat equation on $M_{\infty} \times$ $(0, T)$ limiting to the $\delta$-function centered at $p$ as time approaches $T$.

If we suppose the injectivity radius at $x_{k}$ with the metric $g_{k}(0)$ is uniformly bounded from below, then there exists a subsequence of $\left(M_{k}, g_{k}(t), x_{k}\right)$ which converges to a pointed complete solution to the Ricci flow $\left(M_{\infty}, g_{\infty}(t), x\right)$ by the compactness result of Ricci flow [2, 4].

This convergence result is crucially needed in the proof of Perelman's pseudolocality theorem ([8], Section 10). Perelman states the claim without proof. There are statements and proofs of this convergence result in [6] (Section 33), [1] (Lemma 8.1), as well as in [5] (Theorem 2.1). The first two do not provide complete details, but we had a problem understanding [5]. In this article, we will give the complete details of Theorem 0.1 .

\section{Estimates of the minimal positive fundamental solutions}

In this section, we will list some results about the estimates of the fundamental solutions from [1. In 7, P. Li and S.T. Yau obtained some good estimates of the fundamental solutions without Ricci flow. In this section, we only consider a complete smooth solution $(M, g(t))(\operatorname{dim} M=n)$ of Ricci flow with bounded curvature, $t \in[0, T], T<\infty$.

Definition 1.1. Suppose $(M, g(t))(t \in[0, T])$ is a solution to the Ricci flow on a complete non-compact manifold with bounded curvature. We say that $H(p, T ; x, t)$ is a fundamental solution of the conjugate heat equation centered at $(p, T)(p \in M)$ if

$$
\left\{\begin{aligned}
\left(-\frac{\partial}{\partial t}-\triangle+R\right) H(p, T ; x, t) & =0, \\
\lim _{t \rightarrow T} H(p, T ; x, t) & =\delta_{p} .
\end{aligned}\right.
$$

We say that a fundamental solution $H$ to the conjugate heat equation is the minimal positive fundamental solution if $H$ is positive and if for every positive fundamental solution $h$ to (1.1) we have $h \geq H$. 
The minimal positive fundamental solution on the complete non-compact manifold with bounded curvature exists (see for example [3]) and is unique by definition. Let $u(x, t)=H(p, T ; x, t)$ be the minimal positive fundamental solution of $(1.1)$ on $M \times[0, T)$.

Lemma 1.2. Let $u(x, t)$ be as above. Then

$$
\int_{M} u(x, t) d V_{t}(x)=1
$$

for any $t \in[0, T)$, where $d V_{t}(x)$ denotes the volume form with the metric $g(t)$.

Proof. The proof of this lemma is similar to the proof of Lemma 5.1 in [1].

Corollary 5.2 in [1] states

Lemma 1.3. There exist positive constants $C$ and $D$ with $C$ depending only on $T, n$, and the upper bound of $\left|R_{i j}\right|$, and $D$ depending only on $T$ and the upper bound of $\left|R_{i j}\right|$, such that

$$
u(x, t) \leq \frac{C}{V_{p}(\sqrt{T-t})} e^{-\frac{r^{2}(x)}{D(T-t)}}
$$

for any $t \in[0, T)$, where $r(x)$ is the distance function from $x$ to $p$ with the initial metric $g(0)$ and $V_{p}(\sqrt{T-t})$ is the volume of the ball of radius $\sqrt{T-t}$ at center $p$ with the initial metric $g(0)$.

Lemma 1.4. Suppose $\left|R_{i j}\right| \leq(n-1) K, K$ is a positive constant. Then there is a positive constant $C$ depending only on $n, T, K, D$ ( $D$ is the same as in Lemma 1.3) such that

$$
\int_{B_{p}(A \sqrt{T-t})} u(x, t) d V_{t}(x) \geq 1-C e^{-\frac{A^{2}}{D}}
$$

holds for all $A \geq C$ and all $t \in[0, T)$.

Proof. The proof is similar to the proof of Lemma 5.5 in [1. For the sake of completeness, we will give the proof. By Lemma 1.3,

$$
\begin{aligned}
& \int_{M \backslash B_{p}(A \sqrt{T-t})} u(x, t) d V_{t}(x) \\
\leq & \frac{C_{1}}{V_{p}(\sqrt{T-t})} \int_{M \backslash B_{p}(A \sqrt{T-t})} e^{-\frac{r^{2}(x)}{D(T-t)}} d V_{p}(x) \\
= & \frac{C_{1}}{V_{p}(\sqrt{T-t})} \int_{A \sqrt{T-t}}^{\infty} e^{-\frac{r^{2}}{D(T-t)}} d V_{p}(r) \\
\leq & C_{1} \int_{A \sqrt{T-t}}^{\infty} \frac{V_{p}(r)}{V_{p}(\sqrt{T-t})} \times e^{-\frac{r^{2}}{D(T-t)}} d\left(\frac{r^{2}}{D(T-t)}\right),
\end{aligned}
$$

where $V_{p}(r)$ denotes the volume of the ball $B_{p}(r)$ with the initial metric $g(0)$ and $C_{1}$ depends on the constant $C$ in Lemma 1.3 and $K, T$. The first inequality has used the Ricci flow equation and $\left|R_{i j}\right| \leq(n-1) K$, so the metrics $g(0)$ and $g(t)$ are equivalent; the last inequality has used the integration by parts.

W.l.o.g. assume $C \geq 1$. Then $r \geq A \sqrt{T-t} \geq C \sqrt{T-t}$, by volume comparison theorem [9],

$$
\frac{V_{p}(r)}{V_{p}(\sqrt{T-t})} \leq \frac{V_{K}(r)}{V_{K}(\sqrt{T-t})}
$$


where $V_{K}(r)$ denotes the volume of a ball of radius $r$ in the constant curvature $-K$ space form, and we have

$$
V_{K}(r)=\int_{0}^{r}\left(\frac{\sinh (\sqrt{K} s)}{\sqrt{K}}\right)^{n-1} d s \cdot n \omega_{n},
$$

where $\omega_{n}$ denotes the volume of the $n$-dimensional Euclidean unit ball. Since $\cosh t \geq 1$ and $\frac{d}{d t}\left(\sinh ^{n} t\right)=n \cosh t \sinh ^{n-1} t$, so

$$
V_{K}(r) \leq \frac{\sinh ^{n}(\sqrt{K} r) \omega_{n}}{(\sqrt{K})^{n}}
$$

and

$$
\begin{aligned}
\frac{\sinh (\sqrt{K} r)}{r} & \leq \sqrt{K} e^{\sqrt{K} r} \\
\sinh r & >r
\end{aligned}
$$

for all $r>0$. So by (1.6)-(1.9), we have

$$
\frac{V_{p}(r)}{V_{p}(\sqrt{T-t})} \leq\left(\frac{r}{\sqrt{T-t}}\right)^{n} e^{\sqrt{K} n r}
$$

So when $A \geq 2 \sqrt{K} n T D$, by (1.5) we have

$$
\begin{aligned}
& \int_{M \backslash B_{p}(A \sqrt{T-t})} u(x, t) d V_{t}(x) \\
\leq & \frac{C_{1}}{D} \int_{A \sqrt{T-t}}^{\infty}\left(\frac{r}{\sqrt{T-t}}\right)^{n} e^{-\frac{r^{2}}{D(T-t)}+\sqrt{K} n T \frac{r}{\sqrt{T-t}}} d\left(\frac{r^{2}}{T-t}\right) \\
\leq & \frac{C_{1}}{D} \int_{A \sqrt{T-t}}^{\infty}\left(\frac{r}{\sqrt{T-t}}\right)^{n} e^{-\frac{r^{2}}{2 D(T-t)}} d\left(\frac{r^{2}}{T-t}\right) \\
= & \frac{C_{1}}{D} \int_{\frac{A^{2}}{2 D}}^{\infty}(2 D x)^{\frac{n}{2}} e^{-x} d(2 D x) \\
= & 2^{\frac{n}{2}+1} C_{1} D^{\frac{n}{2}} \int_{\frac{A^{2}}{2 D}}^{\infty} x^{\frac{n}{2}} e^{-x} d x \\
\leq & \left.2^{\frac{n}{2}+1} C_{1} D^{\frac{n}{2}} \int_{\frac{A^{2}}{2 D}}^{\infty} e^{-\frac{x}{2}} d x \quad \text { (when } A^{2} \geq 4 D n^{2}\right) \\
= & 2^{\frac{n}{2}+2} C_{1} D^{\frac{n}{2}} e^{-\frac{A^{2}}{4 D}} .
\end{aligned}
$$

Let $C=\max \left\{1,2 \sqrt{K} n T D, 2 \sqrt{D} n, 2^{\frac{n}{2}+2} C_{1} D^{\frac{n}{2}}\right\}$, so by Lemma 1.2 we obtain the result.

From the proof of Lemma 6.1 in [1], we have the following lemma.

Lemma 1.5. Suppose $\left|\nabla^{k} R m\right|$ are uniformly bounded on $M \times[0, T]$ by $c_{k}$ for all $k$. Let $u>0$ be a solution of (0.2) such that $u \leq A$ for all $t$. Then there is a constant $C$ depending only on $c_{0}, c_{1}, n, T$, such that

$$
\tau|\nabla u| \leq C\left(1+A^{2}\right)
$$

for all $(x, t) \in M \times[0, T)$, where $\tau=T-t$. 


\section{The Proof of Theorem 0.1}

Proof. First, we want to prove for every compact set of $M_{\infty} \times(0, T)$ that $\Phi_{k}^{*} u_{k}$ are uniformly bounded when $k$ is large enough.

For every compact set $\overline{B(R)} \times\left[t_{1}, t_{2}\right]$ of $M_{\infty} \times(0, T)$, where $B(R)$ is the ball of radius $R$ at center $p$ with the metric $g_{\infty}(0), 0<t_{1}<t_{2}<T$. Since $\Phi_{k}^{*} g_{k}$ converges to $g_{\infty}$ uniformly on $\overline{B(R)} \times[0, T]$, there exists $N>0$ such that when $k \geq N$,

$$
\begin{aligned}
\overline{B(R)} & \subset U_{k}, \\
\Phi_{k}\left(B\left(\frac{\sqrt{T-t_{2}}}{2}\right)\right) & \subset B\left(x_{k}, \sqrt{\frac{T-t_{2}}{2}}\right) .
\end{aligned}
$$

Here we choose $R$ large enough such that $T<R^{2}$, where $B\left(x_{k}, r\right)$ denotes the ball of radius $r$ at center $x_{k}$ with metric $g_{k}(0)$. By Lemma 1.3, we have

$$
u_{k}(x, t) \leq \frac{C}{V\left(x_{k}, \sqrt{T-t}\right)} \leq \frac{C}{V\left(x_{k}, \sqrt{T-t_{2}}\right)}
$$

for all $(x, t) \in M_{k} \times\left[0, t_{2}\right]$, where $V\left(x_{k}, r\right)$ denotes the volume of ball $B\left(x_{k}, r\right)$ with the metric $g_{k}(0)$.

So by (2.1)-(2.3), when $k \geq N$, we have

$$
\Phi_{k}^{*} u_{k}(x, t) \leq \frac{C}{V_{p}\left(\frac{\sqrt{T-t_{2}}}{2}\right)}
$$

for all $(x, t) \in \overline{B(R)} \times\left[t_{1}, t_{2}\right]$, where $C$ is independent of $k$. Hence we have proved $\Phi_{k}^{*} u_{k}$ are uniformly bounded on $\overline{B(R)} \times\left[t_{1}, t_{2}\right]$ when $k \geq N$.

Since $\left|R m_{k}\right|(x, t) \leq K$, by Shi's estimate [10, there exist constants $c_{m}$ such that

$$
\left|\nabla^{m} R m_{k}\right|(x, t) \leq \frac{c_{m}}{t^{\frac{m}{2}}}, 0<t \leq T .
$$

So $\left|\nabla^{m} R m_{k}\right|(x, t)$ are uniformly bounded on $M_{k} \times\left[t_{1}, \frac{T+t_{2}}{2}\right]$. Now we consider $u_{k}(x, t)$ as a solution of $(0.2)$ on $M_{k} \times\left[t_{1}, \frac{T+t_{2}}{2}\right]$. As in (2.3), we obtain

$$
u_{k}(x, t) \leq A=\frac{C}{V\left(x_{k}, \sqrt{\frac{T-t_{2}}{2}}\right)}
$$

for all $(x, t) \in M_{k} \times\left[0, \frac{T+t_{2}}{2}\right]$. So by Lemma 1.5, we have

$$
\tau\left|\nabla u_{k}\right| \leq C_{1}\left(1+\left(\frac{C}{V\left(x_{k}, \sqrt{\frac{T-t_{2}}{2}}\right)}\right)^{2}\right)
$$

for all $(x, t) \in M_{k} \times\left[t_{1}, \frac{T+t_{2}}{2}\right)$, where $C_{1}$ is the constant $C$ in Lemma 1.5 and $\tau=\frac{T+t_{2}}{2}-t$. So

$$
\left|\nabla u_{k}\right| \leq \frac{2}{T-t_{2}} C_{1}\left(1+\left(\frac{C}{V\left(x_{k}, \sqrt{\frac{T-t_{2}}{2}}\right)}\right)^{2}\right)
$$

for all $(x, t) \in M_{k} \times\left[t_{1}, t_{2}\right]$. Then by (2.1) and (2.2), when $k \geq N$, we have

$$
\left|\nabla \Phi_{k}^{*} u_{k}\right| \leq \frac{2}{T-t_{2}} C_{1}\left(1+\left(\frac{C}{V_{p}\left(\frac{\sqrt{T-t_{2}}}{2}\right)}\right)^{2}\right)
$$

for all $(x, t) \in \overline{B(R)} \times\left[t_{1}, t_{2}\right]$. Hence $\left|\nabla \Phi_{k}^{*} u_{k}\right|$ are uniformly bounded on $\overline{B(R)} \times$ $\left[t_{1}, t_{2}\right]$ when $k \geq N$. 
Similarly, by induction from the proof of Lemma 1.5, we also know for each $l \geq 2$ that $\left|\nabla^{l} \Phi_{k}^{*} u_{k}\right|$ are uniformly bounded on $\overline{B(R)} \times\left[t_{1}, t_{2}\right]$ when $k$ large enough. We will give the outline of the proof.

Suppose $\left|\nabla^{k} u\right| \leq B$ for $0 \leq k \leq m-1$, and let $f_{m}=\left(1+\left|\nabla^{m} u\right|^{2}\right)^{\frac{1}{2}}$. Then there exist positive constants $E_{1}, E_{2}$ depending on $n, m, T, K, c_{1}, \cdots, c_{m-1}$, such that

$$
\begin{gathered}
\left(\triangle-\frac{\partial}{\partial \tau}\right) f_{m} \geq-E_{1} f_{m}, \\
\left(\triangle-\frac{\partial}{\partial \tau}\right)\left|\nabla^{m-1} u\right|^{2} \geq 2 f_{m}^{2}-E_{2} .
\end{gathered}
$$

Let $Q=\tau f+\frac{1}{2}\left(E_{1}+1\right)\left|\nabla^{m-1} u\right|^{2}-E_{2} \tau-\frac{1}{2}\left(E_{1}+1\right) B^{2}$. Then at $\tau=0, Q \leq 0$, and $\left(\triangle-\frac{\partial}{\partial \tau}\right) Q \geq 0$, we also know that there exists a positive constant $E_{3}$ depending on $n, m, T, K, c_{1}, \cdots, c_{m-1}$, such that

$$
\left\langle\left(\triangle-\frac{\partial}{\partial \tau}\right) \nabla^{m-1} u, \nabla^{m-1} u\right\rangle \geq-E_{3} .
$$

Then using a cutoff argument and the fact that $\left|\nabla^{m-1} u\right|$ is bounded, we can obtain

$$
\int_{0}^{T} \int_{M} \exp \left(-a r_{t}^{2}\right) Q^{2} d V_{t} d t<\infty
$$

Then for a maximum principle (Lemma 6.2 in [1) we obtain $Q \leq 0$. So $\tau f_{m}$ is bounded. Hence we obtain for each $l \geq 2$ that $\left|\nabla^{l} \Phi_{k}^{*} u_{k}\right|$ are uniformly bounded on $\overline{B(R)} \times\left[t_{1}, t_{2}\right]$ when $k$ large enough.

Since $\Phi_{k}^{*} g_{k}$ uniformly converges to $g_{\infty}$ on every compact set of $M_{\infty} \times[0, T], \Phi_{k}^{*} u_{k}$ subconverges on $M_{\infty} \times(0, T)$ to a solution $u$ of $(0.2)$. For each $k, \Phi_{k}^{*} u_{k}$ is positive, and by Lemma 1.4 and the maximum principle we have $u>0$ on $M_{\infty} \times(0, T)$.

Next we want to prove $u$ is a $\delta$-function when $t \rightarrow T$.

For any non-negative $\phi(x) \in \mathcal{C}_{0}^{\infty}\left(M_{\infty}\right)$ there exist $R>0, N_{1}>0$, such that

$$
\begin{gathered}
B(R) \subset U_{i}, i \geq N_{1}, \\
\Omega=\operatorname{supp} \phi(x) \subset B(R), \\
\Omega_{i}=\Phi_{i}(\Omega), \\
\phi_{i}(x)=\phi\left(\Phi_{i}^{-1}(x)\right), x \in V_{i} \text { and } \phi_{i}(x)=0, x \in M_{i} \backslash V_{i} .
\end{gathered}
$$

Then

$$
\phi_{i}\left(x_{i}\right)=\phi(p) .
$$

For any $\varepsilon>0$, we choose $t$ such that $T-\left(\frac{\varepsilon}{C_{4}}\right)^{2} \leq t<T$; here $C_{4}$ is the constant $C$ in Lemma 1.4. Let $A$ in Lemma 1.4 equal $\frac{\varepsilon}{\sqrt{T-t}}$, so we have

$$
\int_{B\left(x_{k}, \varepsilon\right)} u_{k}(x, t) d V_{t}^{i}(x) \geq 1-C_{4} e^{-\frac{\varepsilon^{2}}{D(T-t)}}
$$

where $d V_{t}^{i}$ denotes the volume form with the metric $g_{i}(t)$.

Since $\Phi_{k}^{*} g_{k}$ uniformly converges to $g_{\infty}$ on every compact set of $M_{\infty} \times[0, T]$, there exists $N_{2}=N_{2}(\varepsilon) \geq N_{1}$ such that for any $k \geq N_{2}$ we have

$$
\Phi_{k}(B(2 \varepsilon)) \supset B\left(x_{k}, \varepsilon\right) .
$$


So when $i \geq N_{2}$,

$$
\begin{aligned}
& \int_{\Omega} \Phi_{i}^{*} u_{i}(x, t) \phi(x) d V_{\Phi_{i}^{*} g_{i}(t)} \\
= & \int_{\Omega_{i}} u_{i}(x, t) \phi_{i}(x) d V_{t}^{i} \\
= & \int_{\Omega_{i} \backslash B\left(x_{i}, \varepsilon\right)} u_{i}(x, t) \phi_{i}(x) d V_{t}^{i}+\int_{B\left(x_{i}, \varepsilon\right)} u_{i}(x, t) \phi_{i}(x) d V_{t}^{i} \\
\leq & \frac{C_{3} e^{-\frac{\varepsilon^{2}}{D(T-t)}}}{V_{p}(\sqrt{T-t})} \max _{x \in \overline{\Omega_{i}}} \phi_{i}(x) V_{t}^{i}\left(\Omega_{i}\right)+\sup _{x \in B\left(x_{i}, \varepsilon\right)} \phi_{i}(x) \\
\leq & \frac{C_{3} e^{-\frac{\varepsilon^{2}}{D(T-t)}}}{V_{p}(\sqrt{T-t})} \max _{x \in \bar{\Omega}} \phi(x) V_{\Phi_{i}^{*} g_{i}(t)}(\Omega)+\sup _{x \in B(2 \varepsilon)} \phi(x) \\
\leq & C_{5} \frac{C_{3} e^{-\frac{\varepsilon^{2}}{D(T-t)}}}{V_{p}(\sqrt{T-t})} \max _{x \in \bar{\Omega}} \phi(x) V_{g_{\infty}(0)}(\Omega)+\sup _{x \in B(2 \varepsilon)} \phi(x) \\
\leq & C_{5} \frac{C_{3} e^{-\frac{\varepsilon^{2}}{D(T-t)}}}{V_{p}(\sqrt{T-t})} \max _{x \in \bar{\Omega}} \phi(x) V_{g_{\infty}(0)}(B(R))+\sup _{x \in B(2 \varepsilon)} \phi(x) .
\end{aligned}
$$

The first inequality has used Lemma 1.2 and Lemma 1.3; the second inequality has used (2.10); the third inequality holds since $\Phi_{i}^{*} g_{i}$ uniformly converges to $g_{\infty}$ and the metrics $g_{\infty}(t)$ and $g_{\infty}(0)$ are equivalent, $C_{3}$ is the constant $C$ in Lemma 1.3, and $C_{5}$ is a positive constant depending on $T, K, N_{2}$.

On the other hand, when $i \geq N_{2}$,

$$
\begin{aligned}
& \int_{\Omega} \Phi_{i}^{*} u_{i}(x, t) \phi(x) d V_{\Phi_{i}^{*} g_{i}(t)} \\
= & \int_{\Omega_{i}} u_{i}(x, t) \phi_{i}(x) d V_{t}^{i} \\
\geq & \int_{B\left(x_{i}, \varepsilon\right)} u_{i}(x, t) \phi_{i}(x) d V_{t}^{i} \\
\geq & \inf _{x \in B\left(x_{i}, \varepsilon\right)} \phi_{i}(x) \int_{B\left(x_{i}, \varepsilon\right)} u_{i}(x, t) d V_{t}^{i} \\
\geq & \inf _{x \in B(2 \varepsilon)} \phi(x)\left(1-C_{4} e^{-\frac{\varepsilon^{2}}{D(T-t)}}\right) .
\end{aligned}
$$

The last inequality has used Lemma 1.4, (2.9) and (2.10).

By $(2.11),(2.12)$ and $\Phi_{i}^{*} u_{i}, \Phi_{i}^{*} g_{i}$ uniformly converge to $u, g_{\infty}$ on $\overline{B(R)} \times[0, t]$ as $i \rightarrow \infty$, respectively. Letting $i \rightarrow \infty$, we have

$$
\begin{aligned}
\inf _{x \in B(2 \varepsilon)} \phi(x)\left(1-C_{4} e^{-\frac{\varepsilon^{2}}{D(T-t)}}\right) \leq & \int_{\Omega} u(x, t) \phi(x) d V_{t} \\
\leq & C_{5} \frac{C_{3} e^{-\frac{\varepsilon^{2}}{D(T-t)}}}{V_{p}(\sqrt{T-t})} \max _{x \in \bar{\Omega}} \phi(x) V_{g_{\infty}(0)}(B(R)) \\
& \quad \sup _{x \in B(2 \varepsilon)} \phi(x)
\end{aligned}
$$

where $d V_{t}$ denotes the volume form with the metric $g_{\infty}(t)$. 
Let $t \rightarrow T$. Then by (2.13) we have

$$
\inf _{x \in B(2 \varepsilon)} \phi(x) \leq \liminf _{t \rightarrow T} \int_{\Omega} u(x, t) \phi(x) d V_{t} \leq \limsup _{t \rightarrow T} \int_{\Omega} u(x, t) \phi(x) d V_{t} \leq \sup _{x \in B(2 \varepsilon)} \phi(x) .
$$

By the continuity of $\phi(x)$, let $\varepsilon \rightarrow 0$, so

$$
\lim _{t \rightarrow T} \int_{\Omega} u(x, t) \phi(x) d V_{t}=\phi(p),
$$

i.e.

$$
\lim _{t \rightarrow T} \int_{M_{\infty}} u(x, t) \phi(x) d V_{t}=\phi(p) .
$$

By Lemma $1.2, \Phi_{i}^{*} u_{i}, \Phi_{i}^{*} g_{i}$ uniformly converge to $u, g_{\infty}$ on compact sets in $M_{\infty}$ respectively. It's easy to know that for any $0<t_{1}<T$, we have

$$
\int_{M_{\infty}} u(x, t) d V_{t} \leq 1, \forall t \in\left[0, t_{1}\right]
$$

Then by Lemma 1.4, we obtain

$$
\int_{M_{\infty}} u(x, t) d V_{t}=1
$$

So $u$ is the minimal positive fundamental solution to $(0.2)$ on $M_{\infty} \times(0, T)$. We complete the proof of Theorem 0.1.

\section{ACKNOWLEDGEMENTS}

The author would like to thank his advisor, Professor Fuquan Fang, for encouragement and constant help. He would also like to thank his advisor at UCSD, Professor Lei $\mathrm{Ni}$, for encouragement and many useful discussions, and Professor Ben Chow for discussions. The author would also like to thank the referee for pointing out the errors in the initial version of this paper.

\section{REFERENCES}

1. A. Chau, L. F. Tam and C. Yu, Pseudolocality for the Ricci flow and applications, arxiv:math.DG/0701153.

2. B. Chow, P. Lu and L. Ni, Hamilton's Ricci flow, Grad. Stud. Math., 77, Amer. Math. Soc., Providence, RI; Science Press, New York, 2006. MR2274812 (2008a:53068)

3. C. M. Guenther, The fundamental solution on manifolds with time-dependent metrics, J. Geometric Analysis 12 (2002), no. 3, 425-436. MR.1901749 (2003a:58034)

4. R. Hamilton, A compactness property for solutions of the Ricci flow, Amer. J. Math. 117 (1995), no. 3, 545-572. MR1333936 (96c:53056)

5. S. Hsu, Maximum principle and convergence of fundamental solutions for the Ricci flow, arxiv:math.DG/0711.1236.

6. B. Kleiner and J. Lott, Notes on Perelman's papers, Geom. Topol. 12 (2008), no. 5, 2587-2855. MR 2460872

7. P. Li and S.-T. Yau, On the parabolic kernel of the Schrödinger operator, Acta Math. 156 (1986), no. 3-4, 153-201. MR834612 (87f:58156)

8. G. Perelman, The entropy formula for the Ricci flow and its geometric applications, arxiv:math.DG/0211159. 
9. P. Petersen, Riemannian Geometry (Second Edition), Grad. Texts Math., 171, SpringerVerlag, New York, 2006. MR.2243772 (2007a:53001)

10. W.-X. Shi, Deforming the metric on complete Riemannian manifolds, J. Differential Geom. 30 (1989), no. 1, 223-301. MR.1001277(90i:58202)

Chern Institute of Mathematics, Nankai University, Tianjin, People's Republic of CHina

Current address: Department of Mathematics, University of California at San Diego, La Jolla, California 92093

E-mail address: shijin_zhang@yahoo.com 